\title{
The Underrated Circadian System and Its Contribution to Neurodegeneration in Mood Disorders
}

\author{
Rüdiger Hardeland*
}

University of Goettingen, Johann Friedrich Blumenbach Institute of Zoology and Anthropology, Goettingen, Germany

Article Info

\section{Article Notes}

Received: April 03, 2018

Accepted: May 31, 2018

\section{${ }^{*}$ Correspondence:}

Dr. Rüdiger Hardeland, University of Goettingen, Johann

Friedrich Blumenbach Institute of Zoology and Anthropology, Buergerstrasse 50, D-37073 GOETTINGEN, Germany;

Telephone: +49551395414

E-mail: rhardel@gwdg.de

(c) 2018 Hardeland R. This article is distributed under the terms of the Creative Commons Attribution 4.0 International License.

\section{Keywords}

Aging

Bipolar disorder

Circadian

Depression

Melatonin

Neurodegeneration

Seasonal affective disorder

Sirtuins

\section{ABSTRACT}

In the traditional view of mood disorders, the circadian system has been insufficiently considered. However, polymorphisms of circadian oscillator genes in bipolar disorder, seasonal affective disorder, and subforms of major depressive disorder as well as demonstrable deviations in overt circadian rhythms indicate a role of the circadian system in these pathologies. Circadian malfunction affects sleep, and sleep deprivation can initiate proinflammatory responses. Being parts of the circadian system, melatonin and sirtuin 1 deserve particular attention. Either of them displays neuroprotective, antiinflammatory, and circadian amplitude-enhancing properties, which are of relevance to neurodegeneration that is observed in a number of depressive patients. Notably, both circulating melatonin levels as well as sirtuin 1 expression decline by age. In the gerontological context, melatonin upregulates sirtuin 1, which mediates some of melatonin's actions. Correction of a deviating circadian system seems to be of value regarding causes that contribute to depressive symptoms.

\section{Introduction}

Mood disorders had been regarded in the past largely from a psychological point of view. However, a growing body of evidence indicated that changes in brain physiology are of eminent importance to the development of these pathologies. This was for a while mainly seen in the context of neurotransmitter availability in relevant brain structures and, according to this context, actions of antidepressants were interpreted and developed. The suspicion that neurodegeneration may play a crucial role is not entirely new, but has gained increasing attention mainly during the last years ${ }^{1-6}$. In the beginning, this seemed more likely in cases in which patients exhibited socially unacceptable behavior or signs of dementia, ${ }^{7-10}$ but was less considered in exclusively depressive disorders, a view that is actually changing ${ }^{2,4}$. On the one hand, reduced neurotransmitter concentrations in the synaptic cleft may already be associated with the initiation of neurodegenerative processes, as judged from the influence of other synaptopathic changes, ${ }^{10,11}$ and from the fact that classic antidepressants can also display neuroprotective properties $^{12}$. Therefore, a functional bridge seems to exist between earlier and more recent concepts. On the other hand, numerous other factors can also contribute to neurodegeneration and, thereby, promote mood disorders. In this context, chronic stress ${ }^{13}$ and lowgrade neuroinflammation seem to be of particular importance ${ }^{14-17}$. As mood disorders are typically associated with sleep disturbances that may even be prodromal to depressive symptoms, ${ }^{18-20}$ consequences 
of sleep loss and inappropriate circadian phasing of sleep have to be considered in the etiology of mood disorders.

\section{Consequences of Sleep Disturbances}

Sleep disturbances are a cause of stress and the resulting allostatic load has been implicated in the development of depressive disorders ${ }^{21}$. More importantly, the induction of low-grade inflammation by sleep deprivation has been recognized as a pathological consequence that has the potential for causing various morbidities, especially by inducing low-grade inflammation ${ }^{22-26}$. Low-grade oxidative stress and neuroinflammation typically precede clinical symptoms of neurodegeneration ${ }^{27}$. Details concerning the relationship between oxidative/nitrosative stress, inflammation and neurodegeneration have been outlined and reviewed elsewhere ${ }^{28-30}$. Numerous other findings that have been repeatedly reviewed, meta-analyzed or studied on a broad scale have shown that sleep loss can be associated with depressive disorders ${ }^{18,19,31-35}$ and neurodegeneration ${ }^{4,36-38}$. Although the causality has often remained unclear, inflammatory processes seem to be frequently involved in the initiation of these pathologies. Amyloid- $\beta$ peptides, which contribute to neuroinflammation, have been shown to be increased by sleep loss in the CSF of healthy subjects ${ }^{39,40}$. Sleep disturbances can have different causes, but they are often related to changes in the circadian system. In this context, it is necessary to distinguish between deviations because of gene variants concerning components of the cellular oscillator machinery and age-associated changes that may result from neurodegeneration, epigenetic dysregulation or decreased expression of amplitude-enhancing factors such as sirtuin 1 (SIRT1) or melatonin ${ }^{4,28}$. Moreover, melatonin is also a sleep-promoting agent, especially in terms of initiating sleep onset ${ }^{41,42}$. Polymorphisms of core oscillator components such as the Period 3 (Per3) gene have been associated with inflammation ${ }^{43}$. In turn, inflammation can also reduce the expression of Per2 (Period 2), ${ }^{44}$ presumably by an epigenetic mechanism. Additionally, the anti-inflammatory properties of the accessory oscillator component SIRT1 and the circadian regulator molecule melatonin have to be taken into account ${ }^{28,29}$. Notably, melatonin has been repeatedly shown to upregulate SIRT1 expression, when acting in the context of inflammation, especially neuroinflammation, and of aging, in contrast to its downregulation in cancer cells, as summarized elsewhere $28,29,45$.

\section{Circadian Malfunction in Depressive Disorders}

The assumption of a relevant role of circadian malfunction in the development of mood disorders has received considerable support by two lines of evidence, (i) the association of circadian gene polymorphisms or underexpression with various forms of depressive disorders, and (ii) the demonstration of rhythm deviations in depressive patients. As summarized elsewhere, ${ }^{4,46}$ the genetic relationship was reported for the following types of depression and oscillator genes: bipolar disorder (BP) Per3, Cry2 (cryptochrome 2), Bmal1 (brain and muscle aryl hydrocarbon receptor nuclear translocator-like protein 1 ), Bmal2, Clock (circadian locomotor output cycles kaput), Dbp (D-box binding PAR bZIP transcription factor), Tim

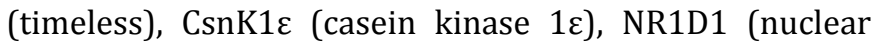
receptor subfamily 1 group D member 1 ); seasonal affective disorders (SAD) - Per2, Cry2, Bmal1, Npas2 (neuronal PAS domain protein 2); subtypes of major depressive disorder (MDD) - Per3, Cry1, Clock, Npas2. Determinations of time-of-death expression in the brains of MDD patients revealed altered expression levels of various oscillator genes, such as Per1, Per2, Per3, Bmal1, NR1D1, Dbp, Dec1 (deleted in esophageal cancer 1) and Dec2 ${ }^{47}$. Because of its amplitude-enhancing properties, SIRT1 is of particular importance and, again, a polymorphism in the Sirt1 gene was demonstrated in $\mathrm{MDD}^{48}$. Additionally, polymorphisms of Per1 and Clock or Per1 and Npas2 were found to be associated with other behavioral disorders, such as attention deficit hyperactivity disorder (ADHD) and autism spectrum disorders (ASD), respectively ${ }^{46}$. Collectively, these data strongly support a contribution of deviations in the circadian system to the etiologies of mood disorders. Notably, various of these oscillator genes, in particular, Per1, Per2, Bmal1, Clock, Npas2 and Sirt1, have been found to be modulated by melatonin ${ }^{45,46,49,50}$. Insofar, it is not surprising that polymorphisms of genes involved in melatonin synthesis and signaling were also reported to be associated with depressive disorders ${ }^{46,51}$. Decreases in melatonin levels also occur in response to epigenetic changes or other pathology-induced effects on the pineal gland ${ }^{52}$ and, thus, can be independent of genetic deficiencies. Nevertheless, the polymorphisms are well in accordance with decreased melatonin levels observed in patients with depressive disorders, as recently summarized ${ }^{4}$.

Of course, a single association does not signify more than just a risk factor, and some of the mutant alleles have also been found in the general population. Nevertheless, the relevance of circadian deviations in depressive disorders is amply documented. The disruption of circadian oscillator functions in depression has been recently discussed ${ }^{4}$. Such findings were also demonstrated in post-mortem brain tissue: while core clock gene expression exhibited robust $24 \mathrm{hr}$-rhythms in six brain regions of control subjects, the oscillations of 11 clock genes were significantly disrupted in MDD, as shown in 34 patients $^{53}$. In the brains of depressive patients, structural changes were clearly apparent, especially in the anterior cingulate cortex, in which the volume of grey matter was reduced ${ }^{53,54}$. Because of its connections to hippocampus, amygdala and striatum, changes in this area are believed to extend to other 
functions. An important aspect concerning the circadian system in depressive disorders concerns the deviations in period length ${ }^{55,56}$. These may be longer or shorter than in the general population, an observation that is by no means contradictory, but rather reflects the difficulties of the organism to be fully synchronized with the $24 \mathrm{~h}$ environment under either of these conditions, as soon as the so-called range of entrainment is exceeded. As the circadian system is not only based on a single master clock, but is composed of numerous central and peripheral oscillators, this can also lead to difficulties of internal synchrony between rhythms that depend to a variable degree on the hypothalamic master clock, the suprachiasmatic nucleus (SCN) and may be semi-autonomous or, in the extreme, more or less autonomous with regard to the $\mathrm{SCN}^{45,46}$. Misalignment of rhythms both in relation to external time cues and internally within the body may contribute to physiological problems, which, in the end, may turn into difficulties of sleep and mood disorders. From this point of view, it would be of substantial importance to first assess the extent and direction of circadian deviations in a depressive patient and, thereafter, to select treatments that allow a forced synchronization by either shortening or lengthening the circadian periods ${ }^{4,56}$. Possibilities of using melatonin or synthetic melatonergic agonists and/ or bright light in appropriate circadian phases have been discussed elsewhere ${ }^{4,51}$.

\section{An Antidepressant May Affect Circadian Oscillations}

A specific point is worth being addressed herein, namely the influence of antidepressants on the circadian system. Several anti-depressive drugs, especially serotonin reuptake inhibitors, are capable of disturbing sleep and may require concomitant treatment with sedative/hypnotics, ${ }^{57-59}$ whereas other antidepressants with sedative properties such as doxepin, mirtazapine, and trazodone rather promote sleep $^{59}$. Sleep disturbances by pharmacological treatments may have secondary effects on the circadian system, and the important question is that of whether these alterations may change the circadian oscillations towards normality or drive them towards poorer entrainment. Moreover, an antidepressant can have a direct effect on circadian period lengths. This is particularly the case with lithium, which is known since decades to lengthen circadian oscillations. ${ }^{4}$ Therefore, lithium will beneficial in a patient who is poorly synchronized because of a pathologically shortened period length, but counterproductive in another patient with an extended period, as has been shown in patients with bipolar disorder ${ }^{4,60,61}$.

Depressive Disorders, Neurodegeneration and the Protective Role of Melatonin

While circadian changes are frequent in depressive disorders, the link to neurodegeneration requires further elucidation. Generally, one has to remain aware that neurodegeneration is not a diagnostic criterion for depression. On the one hand, neurodegenerative processes can induce malfunction of the circadian system, ${ }^{28,29}$ cause sleep disturbances and, thereby, may also promote the development of depressive disorders. On the other hand, damage to brain regions may presumably also occur as a consequence of persisting alterations by circadian misalignment. Sleep loss has been shown to be associated with brain atrophy, as demonstrated by an expansion of ventricular volume ${ }^{62}$. Moreover, reductions in in the levels of neuroprotective agents such as melatonin and SIRT1 may also facilitate neurodegenerative processes. Notably, these two molecules are important components of the circadian system. Therefore, decreases in melatonin and SIRT1, which mediates, at least, some of melatonin's actions, ${ }^{45,63}$ reflect a weakening of the circadian system. Additionally, they indicate insufficient stimulation of circadian oscillators by these two important amplitude-enhancing factors. Moreover, both melatonin and SIRT1 display antiinflammatory properties, ${ }^{28,29,64-66}$ and have been shown to be neuroprotective ${ }^{29,51,67}$. Many details are known on antioxidative/antinitrosative actions of melatonin ${ }^{30,68-70}$. Notably, antioxidative protection is strongly related to the circadian system, which includes but also exceeds the rhythmicity of melatonin ${ }^{45,71}$.

The protective roles of melatonin have been analyzed many times under various aspects and comprise various direct and indirect antioxidant, mitochondria protecting and anti-excitatory actions, which also interrupt vicious cycles that emerge from cross-talks between neurons, astrocytes and microglia ${ }^{28-30,72}$. Similar assumptions may be deduced for SIRT1, but this would require more extended documentation. Despite the necessity of further exploring the connections between circadian malfunction and neurodegeneration, the degenerative changes in depressive disorders have been amply described. Decreases in the volumes of brain regions have been shown in MDD, especially concerning the prefrontal, more strongly, the orbital frontal, and the anterior cingulate cortex, caudate nucleus, putamen, hippocampus and, occasionally, also other brain regions ${ }^{4}$. With regard to the inflammatory aspects of BP, such as increases in proinflammatory cytokines and markers, ${ }^{4}$ neurodegenerative processes are likely promoted in this form of depression, too. This would also conform with similar observations in sleep disturbances $^{28}$. Further studies on neurodegeneration after a long-lasting BP history should clarify this important perspective.

\section{Conclusion}

With regard to the high incidence of depressive disorders that affect more than 120 million people 
worldwide, with a rising tendency and an established impact concerning working disability, morbidity and mortality, ${ }^{18}$ the causes and etiological relationships of these pathologies deserve particular attention and further research. Concerning the role of melatonin on depressive disorders, it is important to remain aware that this compound is not a direct antidepressant. Therefore, is has not been a surprise that a recent systematic metaanalysis has not revealed demonstrable improvements by melatonin in depressive disorders ${ }^{73}$. However, it will be important to study the effects of melatonin in the context of circadian readjustments, which should have effects on sleep, anti-inflammatory and other neuroprotective actions that can be expected to counteract depression-promoting changes. This has not yet been sufficiently done and the chronobiological rules for re-entrainment of rhythms have mostly been disregarded. Moreover, neurodegenerative processes represent long-term developments that cannot be rapidly reversed. Neurodegeneration is, in the understanding of this author, a major concern. The increasing evidence for proinflammatory changes by sleep deprivation and the involvement of circadian deviations in mood disorders indicate a necessity for more strongly considering malfunction of the circadian system and its consequences to sleep in such a context. Of course, one has to remain aware of the heterogeneity of depressive disorders, in which even MDD turns out to be a complex of different pathological subforms with their own etiologies. Nevertheless, the common symptom of sleep disturbance is already a sufficient reason for looking closely at low-grade neuroinflammation and pertinent changes such as microglia activation, astrocytic and neuronal damage. Moreover, the association of the incidence of depressive disorders with age, which is anyway related to proinflammatory traits by immunosenescence, senescence-associated secretory phenotype, brain insulin resistance and increased levels of amyloid- $\beta$ peptides and oligomers, ${ }^{27,32,49,50}$ leads to the necessity for particular attention in an aging society. On the side of interventions, the correction of circadian malfunction, e.g., by appropriately timed melatonin and bright light appears to be an option that may deserve more consideration. ${ }^{4}$ Either of these treatments is extremely safe, compared to the use of most classic antidepressants. Additionally, melatonin's other beneficial actions as an antioxidant, anti-inflammatory agent, neuroprotectant and stimulator of SIRT1 expression should be taken into account ${ }^{32,33,49}$. However, it will be important to determine the specific circadian deviations of an individual patient before schematically applying a chronotherapy ${ }^{4}$. Otherwise, a treatment that further lengthens an already pathologically lengthened circadian period, or shortens an already shortened period, will only lead to wrong conclusions on non-responsiveness.

\section{Conflict of interest}

This author declares no actual or potential conflicts of interest.

\section{References}

1. Weisenbach SL, Kumar A. Current understanding of the neurobiology and longitudinal course of geriatric depression. Curr Psychiatry Rep. 2014; 16(9): 463. doi: 10.1007/s11920-014-0463-y.

2. Shioya A, Saito Y, Arima K, et al. Neurodegenerative changes in patients with clinical history of bipolar disorders. Neuropathology. 2015; 35(3): 245-253. doi: 10.1111/neup.12191.

3. Kalenderoglu A, Çelik M, Sevgi-Karadag A, et al. Optic coherence tomography shows inflammation and degeneration in major depressive disorder patients correlated with disease severity. J Affect Disord. 2016; 204: 159-165. doi: 10.1016/j.jad.2016.06.039.

4. Brown GM, McIntyre RS, Rosenblat J, et al. Depressive disorders: Processes leading to neurogeneration and potential novel treatments. Prog Neuropsychopharmacol Biol Psychiatry. 2018; 80(Pt C): 189 204. doi: 10.1016/j.pnpbp.2017.04.023

5. Khalil MA, Saleh AA, Gohar SM, et al. Optical coherence tomography findings in patients with bipolar disorder. J Affect Disord. 2017; 218: 115-122. doi: 10.1016/j.jad.2017.04.055.

6. Tang SW, Helmeste DM, Leonard BE. Neurodegeneration, neuroregeneration, and neuroprotection in psychiatric disorders. Mod Trends Pharmacopsychiatry. 2017; 31: 107-123. doi: $10.1159 / 000470811$.

7. Desmarais P, Lanctôt KL, Masellis M, et al. Social inappropriateness in neurodegenerative disorders. Int Psychogeriatr. 2018; 30(2): 197 207. doi: $10.1017 /$ S1041610217001260.

8. Fujishiro H, Iseki E, Nakamura S, et al. Dementia with Lewy bodies: early diagnostic challenges. Psychogeriatrics. 2013; 13(2): 128-138. doi: $10.1111 /$ psyg.12005.

9. Munro CE, Donovan NJ, Guercio BJ, et al. Neuropsychiatric symptoms and functional connectivity in mild cognitive impairment. J Alzheimers Dis. 2015; 46(3): 727-735. doi: 10.3233/JAD-150017.

10. Beckman D, Linden R. A roadmap for investigating the role of the prion protein in depression associated with neurodegenerative disease. Prion. 2016; 10(2): 131-142. doi: 10.1080/19336896.2016.

11. Tyebji S, Hannan AJ. Synaptopathic mechanisms of neurodegeneration and dementia: Insights from Huntington's disease. Prog Neurobiol. 2017; 153: 18-45. doi: 10.1016/j.pneurobio.2017.03.008.

12. Tizabi Y. Duality of antidepressants and neuroprotectants. Neurotox Res. 2016; 30(1): 1-13. doi: 10.1007/s12640-015-9577-1

13. Vyas S, Rodrigues AJ, Silva JM, et al. Chronic stress and glucocorticoids: from neuronal plasticity to neurodegeneration. Neural Plast. 2016; 2016: 6391686. doi: 10.1155/2016/6391686.

14. Hoyo-Becerra C, Schlaak JF, Hermann DM. Insights from interferon$\alpha$-related depression for the pathogenesis of depression associated with inflammation. Brain Behav Immun. 2014; 42: 222-231. doi: 10.1016/j.bbi.2014.06.200.

15. Jeon SW, Kim YK. Neuroinflammation and cytokine abnormality in major depression: Cause or consequence in that illness? World J Psychiatry. 2016; 6(3): 283-293. doi: 10.5498/wjp.v6.i3.283.

16. Singhal G, Baune BT. Microglia: an interface between the loss of neuroplasticity and depression. Front Cell Neurosci. 2017; 11: 270. doi: 10.3389/fncel.2017.00270.

17. Swardfager W, Hennebelle M, Yu D, et al. Metabolic/inflammatory/ vascular comorbidity in psychiatric disorders; soluble epoxide hydrolase ( $\mathrm{sEH})$ as a possible new target. Neurosci Biobehav Rev. 2018; 87: 56-66. doi: 10.1016/j.neubiorev.2018.01.010. 
18. Srinivasan V, Pandi-Perumal SR, Trakht I, et al. Pathophysiology of depression: role of sleep and the melatonergic system. Psychiatry Res. 2009; 165(3): 201-214. doi: 10.1016/j.psychres.2007.11.020.

19. Pandi-Perumal SR, Moscovitch A, Srinivasan V, et al. Bidirectional communication between sleep and circadian rhythms and its implications for depression: lessons from agomelatine. Prog Neurobiol. 2009; 88(4): 264-271. doi: 10.1016/j.pneurobio.2009.04.007.

20. Zaki NFW, Spence DW, BaHammam AS, et al. Chronobiological theories of mood disorder. Eur Arch Psychiatry Clin Neurosci. 2018; 268(2): 107-118. doi: 10.1007/s00406-017-0835-5.

21. McEwen BS. Mood disorders and allostatic load. Biol Psychiatry. 2003; 54(3): 200-207.

22. Vgontzas AN, Zoumakis E, Bixler EO, et al. 2004. Adverse effects of modest sleep restriction on sleepiness, performance, and inflammatory cytokines. J Clin Endocrinol Metab. 2004; 89: 2119-2126.

23. Mullington JM, Simpson NS, Meier-Ewert HK, et al. Sleep loss and inflammation. Best Pract Res Clin Endocrinol Metab. 2010; 24: 775784. doi: 10.1016/j.beem.2010.08.014.

24. Rosa Neto JC, Lira FS, Venancio DP, et al. Sleep deprivation affects inflammatory marker expression in adipose tissue. Lipids Health Dis. 2010; 9: 125. doi: 10.1186/1476-511X-9-125.

25. Ramesh V, Nair D, Zhang SX, et al. Disrupted sleep without sleep curtailment induces sleepiness and cognitive dysfunction via the tumor necrosis factor- $\alpha$ pathway. J Neuroinflammation. 2012; 9: 91. doi: 10.1186/1742-2094-9-91.

26. Prather AA, Vogelzangs N, Penninx BWJH. Sleep duration, insomnia, and markers of systemic inflammation: Results from the Netherlands Study of Depression and Anxiety (NESDA). J Psychiatr Res. 2015; 60 95-102. doi: 10.1016/j.jpsychires.2014.09.018.

27. Cuadrado A, Manda G, Hassan A, et al. Transcription factor NRF2 as a therapeutic target for chronic diseases: a systems medicine approach. Pharmacol Rev. 2018 70(2): 348-383. doi: 10.1124/pr.117.014753.

28. Hardeland R. Brain inflammaging: roles of melatonin, circadian clocks and sirtuins. J Clin Cell Immunol. 2018; 9: 543. doi: 10.4172/21559899.1000543.

29. Hardeland R, Cardinali DP, Brown GM, et al. Melatonin and brain inflammaging. Prog Neurobiol. 2015; 127-128: 46-63. doi: 10.1016/j. pneurobio.2015.02.001.

30. Hardeland R. Melatonin and the theories of aging: a critical appraisal of melatonin's role in antiaging mechanisms. J Pineal Res. 2013; 55(4): 325-356. doi: 10.1111/jpi.12090.

31. Perlis ML, Giles DE, Buysse DJ, et al. 1997. Self-reported sleep disturbance as a prodromal symptom in recurrent depression. J Affect Disord. 1997; 42(2-3): 209-212. PMID: 9106853.

32. Gillin JC. Are sleep disturbances risk factors for anxiety, depressive and addictive disorders?Acta Psychiatr Scand Suppl. 1998; 393: 3943. PMID: 9777046.

33. Finan PH, Smith MT. The comorbidity of insomnia, chronic pain, and depression: dopamine as a putative mechanism. Sleep Med Rev. 2013; 17(3): 173-183. doi: 10.1016/j.smrv.2012.03.003.

34. Li L, Wu C, Gan Y, et al. Insomnia and the risk of depression: a metaanalysis of prospective cohort studies. BMC Psychiatry. 2016; 16: 375. doi: 10.1186/s12888-016-1075-3.

35. Freeman D, Sheaves B, Goodwin GM, et al. The effects of improving sleep on mental health (OASIS): a randomised controlled trial with mediation analysis. Lancet Psychiatry. 2017; 4(10): 749-758. doi: 10.1016/S2215-0366(17)30328-0.

36. Musiek ES, Holtzman DM. Mechanisms linking circadian clocks, sleep, and neurodegeneration. Science. 2016; 354(6315): 1004-1008. doi: 10.1126/science.aah4968.
37. Pillai JA, Leverenz JB. Sleep and neurodegeneration: a critical appraisal. Chest. 2017; 151(6): 1375-1386. doi: 10.1016/j.chest.2017.01.002.

38. Zhao Z, Zhao X, Veasey SC. Neural consequences of chronic short sleep: reversible or lasting. Front Neurol. 2017; 8: 235. doi: 10.3389/ fneur.2017.00235.

39. Ooms S, Overeem S, Besse K, et al. Effect of 1 night of total sleep deprivation on cerebrospinal fluid $\beta$-amyloid 42 in healthy middleaged men: a randomized clinical trial. JAMA Neurol. 2014; 71(8): 971 977. doi: 10.1001/jamaneurol.2014.1173.

40. Ju YS, Ooms SJ, Sutphen C, et al. Slow wave sleep disruption increases cerebrospinal fluid amyloid- $\beta$ levels. Brain. 2017; 140(8): 21042111. doi: 10.1093/brain/awx148.

41. Hardeland R. New approaches in the management of insomnia: weighing the advantages of prolonged-release melatonin and synthetic melatoninergic agonists. Neuropsychiatr Dis Treat. 2009; 5: 341-354. doi: 10.2147/NDT.S4234.

42. Hardeland R. Melatonergic sleep promotion: fundamental chronobiological issues concerning sleep onset and maintenance, dose and duration of action. Slee Vigilance. 2018; [Epub ahead of print, Sep 1, 2017]. doi: 10.1007/s41782-017-0014-9.

43. Guess J, Burch JB, Ogoussan K, et al. Circadian disruption, Per3, and human cytokine secretion. Integr Cancer Ther. 2009; 8(4): 329-336. doi: 10.1177/1534735409352029.

44. Lee H, Nah SS, Chang SH, et al. PER2 is downregulated by the LPSinduced inflammatory response in synoviocytes in rheumatoid arthritis and is implicated in disease susceptibility. Mol Med Rep. 2017; 16(1): 422-428. doi: 10.3892/mmr.2017.6578.

45. Hardeland R. Melatonin and the pathologies of weakened or dysregulated circadian oscillators. J Pineal Res. 2017; 62(1): e12377. doi: 10.1111/jpi.12377.

46. Hardeland R, J.A. Madrid JA, Tan DX, et al. Melatonin, the circadian multioscillator system and health: the need for detailed analyses of peripheral melatonin signaling. J Pineal Res. 2012; 52(2): 139-166. doi: 10.1111/j.1600-079X.2011.00934.x.

47. Li JZ, Bunney BG, Meng F, et al. Circadian patterns of gene expression in the human brain and disruption in major depressive disorder. Proc Natl Acad Sci USA. 2013; 110(24): 9950-9955. doi: 10.1073/ pnas.1305814110.

48. Kishi T, Yoshimura R, Kitajima T, et al. SIRT1 gene is associated with major depressive disorder in the Japanese population. J Affect Disord. 2010; 126(1-2): 167-173. doi: 10.1016/j.jad.2010.04.003.

49. Hardeland R. Melatonin and circadian oscillators in aging - A dynamic approach to the multiply connected players. Interdisc Top Gerontol. 2015; 40: 128-140. doi: 10.1159/000364975.

50. Hardeland R. Melatonin, noncoding RNAs, messenger RNA stability and epigenetics - evidence, hints, gaps and perspectives. Int J Mol Sci. 2014; 15(10): 18221-18252. doi: 10.3390/ijms151018221.

51. Hardeland R. Melatonin in aging and disease - multiple consequences of reduced secretion, options and limits of treatment. Aging Dis. 2012; 3(2): 194-225. PMID: 22724080.

52. Markus RP, Fernandes PA, Kinker GS, et al. Immune-pineal axis acute inflammatory responses coordinate melatonin synthesis by pinealocytes and phagocytes. Br J Pharmacol. 2017 [Epub ahead of print, Nov 4]; doi: 10.1111/bph.14083.]

53. Bunney B, Li J, Walsh D, et al. Circadian dysregulation of clock genes: clues to rapid treatments in major depressive disorder. Mol Psychiatry. 2015; 20 (1): 48-55. doi:10.1038/mp.2014.138.

54. Gotlib IH, Sivers H, Gabrieli JD, et al. Subgenual anterior cingulate activation to valenced emotional stimuli in major depression. Neuroreport. 2005; 16(16):1731-1734. PMID: 16237317. 
55. Courtet P, Olié E. Circadian dimension and severity of depression. Eur Neuropsychopharmacol. 2012; 22 Suppl 3: S476-S481. doi: 10.1016/j. euroneuro.2012.07.009

56. Martynhak BJ, Pereira M, de Souza CP, et al. Stretch, shrink, and shatter the rhythms: The intrinsic circadian period in mania and depression. CNS Neurol Disord Drug Targets. 2015; 14(8): 963-969. PMID: 26350335.

57. Pandi-Perumal SR, Trakht I, Srinivasan V, et al. The effect of melatonergic and non-melatonergic antidepressants on sleep: weighing the alternatives. World J Biol Psychiatry. 2009; 10(4 Pt 2): 342-354. doi: 10.1080/15622970701625600.

58. Srinivasan V, Brzezinski A, Pandi-Perumal SR, et al. Melatonin agonists in primary insomnia and depression-associated insomnia: are they superior to sedative-hypnotics. Prog Neuropsychopharmacol Biol Psychiatry. 2011; 35(4): 913-923. doi: 10.1016/j.pnpbp.2011.03.013.

59. Wichniak A, Wierzbicka A, Walęcka M, et al. Effects of antidepressants on sleep. Curr Psychiatry Rep. 2017; 19(9): 63. doi: 10.1007/s11920017-0816-4.

60. Kripke D, Mullaney D, Atkinson M, et al. Circadian rhythm disorders in manic-depressives. Biol Psychiatry. 1978; 13(3): 335-351. PMID: 667233.

61. Moreira J, Geoffroy PA. Lithium and bipolar disorder: Impacts from molecular to behavioural circadian rhythms. Chronobiol Int. 2016; 33(4): 351-373. doi: 10.3109/07420528.2016.1151026.

62. Lo JC, Loh KK, Zheng H, et al. Sleep duration and age-related changes in brain structure and cognitive performance. Sleep 2014; 37(7): 1171-1178. doi: 10.5665/sleep.3832.

63. Yang W, Kang X, Qin N, et al. Melatonin protects chondrocytes from impairment induced by glucocorticoids via $\mathrm{NAD}^{+}$-dependent SIRT1. Steroids. 2017; 126: 24-29. doi: 10.1016/j.steroids.2017.08.005.

64. Hwang JW, Yao H, Caito S, et al. Redox regulation of SIRT1 in inflammation and cellular senescence. Free Radic Biol Med. 2013; 61C: 95-110. doi: 10.1016/j.freeradbiomed.2013.03.015.

65. Hernández-Jiménez M, Hurtado 0 , Cuartero MI, et al. Silent information regulator 1 protects the brain against cerebral ischemic damage. Stroke. 2013; 44(8): 2333-2337. doi: 10.1161/ STROKEAHA.113.001715.

66. Esposito E, Cuzzocrea S. Antiinflammatory activity of melatonin in central nervous system. Curr Neuropharmacol. 2010; 8(3):228-42. doi: 10.2174/157015910792246155.

67. Favero G, Franceschetti L, Bonomini F, et al. Melatonin as an antiinflammatory agent modulating inflammasome activation. Int J Endocrinol. 2017; 2017:1835195. doi: 10.1155/2017/1835195.

68. Hardeland R. Antioxidative protection by melatonin: multiplicity of mechanisms from radical detoxification to radical avoidance. Endocrine. 2005; 27(2): 119-130. PMID: 16217125.

69. Hardeland R, Cardinali DP, Srinivasan V, et al. Melatonin - a pleiotropic, orchestrating regulator molecule. Prog Neurobiol. 2011; 93(3): 350384. doi: 10.1016/j.pneurobio.2010.12.004.

70. Reiter RJ, Mayo JC, Tan DX, et al. Melatonin as an antioxidant: under promises but over delivers. J Pineal Res. 2016; 61(3): 253-278. doi: 10.1111/jpi.12360.

71. Hardeland R, Coto-Montes A, Poeggeler B. Circadian rhythms, oxidative stress, and antioxidative defense mechanisms. Chronobiol Int. 2003; 20(6): 921-962. PMID:14680136.

72. Salminen A, Ojala J, Kaamiranta K, et al. Astrocytes in the aging brain express characteristics of senescence-associated secretory phenotype. Eur J Neurosci. 2011; 34(1): 3-11. doi: 10.1111/j.14609568.2011.07738.x.

73. De Crescenzo F, Lennox A, Gibson JC, et al. Melatonin as a treatment for mood disorders: a systematic review. Acta Psychiatr Scand. 2017; 136(6): 549-558. doi: 10.1111/acps.12755. 